\title{
THE DILOGARITHM IN ALGEBRAIC FIELDS
}

\author{
L. LEWIN \\ (Received 10 August 1981; revised 2 November 1981) \\ Communicated by A. J. van der Poorten
}

\begin{abstract}
Special numerical formulae for the dilogarithm of powers of a base quantity have been shown to exist by Watson, Coxeter and others. Abel's equation for the dilogarithm was put in this "exponent" form. as a result of which a four-variable, symmetrical equation, also in exponent form, was deduced. From these equations a large number of special numerical results were produced, from which certain properties of a general structural nature emerged, and enabled two new results to be predicted.

The algebraic bases for these results can be grouped in trigonometric or non-trigonometric form, and for the former it seems to be necessary to examine the properties of the dilogarithm in the complex plane. Even so, there are some identities that seem to be outside the scope of the present methods.

It is speculated that certain factorization relations, which can be identified from the equations, may play a substantial role in the results; but so far no analytic derivation of this property has appeared possible.
\end{abstract}

1980 Mathematics subject classification (Amer. Math. Soc.): 33 A 70.

\section{Introduction}

There can be few who, having had occasion to study or work with the dilogarithm function, have not encountered some of the peculiar numerical and functional relations it possesses. First studied over two hundred years ago by Euler, Landen and others, it can be defined through the series

$$
\operatorname{Li}_{2}(z)=\sum_{1}^{\infty} z^{n} / n^{2}, \quad|z| \leqslant 1
$$

(C) Copyright Australian Mathematical Society 1982 
or through the integral

$$
\operatorname{Li}_{2}(z)=-\int_{0}^{z} \frac{\log (1-z)}{z} d z
$$

The latter extends the range outside the unit circle, and the function, as thus defined, is real for $z$ real in the range $-\infty<z \leqslant 1$.

The question of definition and notation is discussed extensively in [9]. It should be mentioned here that some authors prefer to work with the function $L(z)$ introduced by Rogers [11] and defined by

$$
L(z)=\mathrm{Li}_{2}(z)+\frac{1}{2} \log (z) \log (1-z) .
$$

This combination has the property of suppressing the presence of logarithmic terms in some of the formulae. However, it is not used here because not only are these logarithmic terms themselves an item of interest, but (3) has the disadvantage of unnecessarily introducing complex forms into the equations when $z$ is negative.

Another function occasionally used was introduced by Legendre [8] and represents the sum of only the odd powers in (1). Using the notation $\chi_{2}(z)$, it is defined by

$$
\chi_{2}(z)=\sum_{0}^{\infty} z^{2 n+1} /(2 n+1)^{2}, \quad|z|<1 .
$$

If these functions possessed little more than a definition and a notation, there would not be much to write about. However, as with the elliptic, Bessel, and other functions of mathematical physics, the dilogarithm possesses an extensive range of formulae involving both single and multiple variables. These functional equations are usually very easy to verify; all one needs to do is to differentiate, which reduces them to simple logarithmic forms via (2). The inverse problem of discovering these relations in the first place is another matter, however, no matter how straightforward it may seem to have been in retrospect. Much the same may be said for some of the special numerical relations which are the subject of this present study, with the added complication that they cannot, in contrast to the functional equations, be verified by a differentiation. Watson [14] indicated that he had long suspected the existence of a certain result, and although his eventual proof is easy enough to follow, it is clear that it was not all that easy to come by. Indeed, we shall indicate shortly two new results of a somewhat similar character, whose form is suggested by general structural considerations. Their correctness has been verified by numerical computation, but, at time of writing, they do not possess any analytical derivation.

Although there is no current proof, there is little doubt that, for $z$ algebraic, $\mathrm{Li}_{2}(z)$ is irrational; and in fact transcendental. Finding a proof is difficult, in 
particular because of the slow convergence of the defining series; although Chudnovsky, through the use of Pade approximations to hypergeometric functions, succeeded in showing that $\mathbf{L i}_{2}$ is irrational for certain algebraic arguments near the origin.

Although transcendental, there are a few special values for which $\mathrm{Li}_{2}$ can be expressed simply in terms of logarithms and $\pi^{2}$, the latter arising, in the present context, via $[\log (-1)]^{2}=-\pi^{2}$. (It is convenient, for this reason, to refer to all such terms as logarithmic.) Euler's results on $\mathrm{Li}_{2}( \pm 1)$ are well known:

(a) $\operatorname{Li}_{2}(1)=\pi^{2} / 6$,

(b) $\quad \operatorname{Li}_{2}(-1)=-\pi^{2} / 12$.

Less well known is his formula

$$
\operatorname{Li}_{2}\left(\frac{1}{2}\right)=\pi^{2} / 12-\frac{1}{2} \log ^{2}(2) .
$$

(We use the convention of writing $\log ^{2}(x)$ to mean $\left[\log _{e}(x)\right]^{2}$.)

Landen [7] investigated functions of the argument

$$
\rho=\frac{1}{2}(\sqrt{5}-1)=2 \cos (2 \pi / 5),
$$

the inverse of the golden ratio $\frac{1}{2}(\sqrt{5}+1)$. He showed that

(a) $\operatorname{Li}_{2}(\rho)+\mathrm{Li}_{2}\left(\rho^{2}\right)=\pi^{2} / 6-2 \log ^{2} \rho$

(b) $\mathrm{Li}_{2}(\rho)-\mathrm{Li}_{2}\left(\rho^{2}\right)=\pi^{2} / 30$,

or

$$
\begin{aligned}
& \text { (a) } \operatorname{Li}_{2}(\rho)=\pi^{2} / 10-\log ^{2} \rho, \\
& \text { (b) } \operatorname{Li}_{2}\left(\rho^{2}\right)=\pi^{2} / 15-\log ^{2} \rho .
\end{aligned}
$$

The above values appear to be the only ones (apart from the inversion of the argument) for which the dilogarithm can be evaluated in non-transcendental form. However, there are many interesting equations relating two or more powers of the argument. Watson's result, referred to earlier, gives a relation very similar to (8a) for the argument $2 \cos (3 \pi / 7)$ and two others, while Coxeter [4], using the properties of a rather involved series, has produced a number of results for powers of $\rho$ which, in a form free of logarithms, can be written

(b) $\quad 4 \mathrm{Li}_{2}\left(\rho^{3}\right)-\mathrm{Li}_{2}\left(\rho^{6}\right)+\mathrm{Li}_{2}\left(\rho^{2}\right)-4 \mathrm{Li}_{2}(\rho)=-\pi^{2} / 6$,

(c) $3 \mathrm{Li}_{2}\left(\rho^{4}\right)+3 \mathrm{Li}_{2}\left(\rho^{6}\right)-\mathrm{Li}_{2}\left(\rho^{12}\right)-3 \operatorname{Li}_{2}\left(\rho^{2}\right)=-\pi^{2} / 15$,

(d) $15 \mathrm{Li}_{2}\left(\rho^{4}\right)+2 \mathrm{Li}_{2}\left(\rho^{10}\right)-\mathrm{Li}_{2}\left(\rho^{20}\right)-10 \mathrm{Li}_{2}\left(\rho^{2}\right)=-\pi^{2} / 5$.

The negative arguments appearing here can all be converted readily into positive ones. One can ask whether Coxeter's or Watson's results are isolated 
cases, or whether there are other relations of the form (8) or (10) for other arguments or indices. Specifically, one can ask for what algebraic numbers $u$ there exist formulae of the form

$$
\mathrm{Li}_{2}\left(u^{N}\right)=\sum_{r=1}^{N-1} A_{r} \mathrm{Li}_{2}\left(u^{r}\right)+A_{0} \pi^{2}+L
$$

where the $A_{r}$ are rational and $L$ is a rational quadratic form in logarithms of rational polynomials of $u$. In this equation the largest exponent $N$ will be referred to as the index of the equation; the quantity $u$ as the base; the indices $r$ as the exponents; and the coefficients $A_{r}$ as the factors. In all but one of the cases to be examined it is found that $L$ reduces to the simple form $B \log ^{2} u$, with $B$ a simple rational. However, the forms taken by the equations themselves do have a somewhat arbitrary aspect. This may be seen from (10), where any linear combination of the equations there could be taken, to create another. Of course, one cannot gain new equations by this route, though one can lose some-for example, (10c) can be shown to be redundant in the sense that it can be obtained fairly readily from (10a) and (12). The question of what combination of equations should be examined is not entirely trivial, however, and it will be shown that, in a number of cases, they can be arranged in a very natural way so that the relation (11) in fact takes on a definite and highly structured form. The index $N$ turns out to be composite, with the exponents $r$ ranging through the factors of $N$, and with the coefficients $A_{r}$ closely related to the complementary factors, that is, to $N / r$. This feature is so striking that it cannot be dismissed as a mere chance result, or as the consequence of a contrived combination of the equations. However, there does not seem to be any obvious explanation at this time, though the property is apparently closely connected with the form taken by the factorization of $\left(1-u^{N}\right)$ in terms of factors $\left(1-u^{r}\right)$ for a particular base $u$. These factorizations are not of a general character but are highly specific to the base, and in fact constitute an equation - albeit of needlessly high degree-for the determination of the base. To each equation of the form (11) there exists a corresponding factorization, though it is not at all true in reverse that to an arbitrary factorization there exists a corresponding equation. This matter is taken a little further in Section 7.

\section{Notation}

We shall be working for the most part with the function $\mathrm{Li}_{2}(z)$ defined in (1) and (2), though $L(z)$ and $\chi_{2}(z)$ of (3) and (4) will occasionally be used.

$k, m, n, p$ and $q$ are used for exponents; generally, but not necessarily, integers.

$x, y, z$ are used for general variables when equations are expressed in algebraic form. 
$u, v, w, U, V, W$, are used for general variables when equations are expressed in exponent form.

Greek lower case letters $\alpha$ to $\omega$ are used to represent specific numerical bases, as defined in later sections. They are all real, positive algebraic numbers less than unity. In a few cases a power of one of these quantities can also represent a base in its own right, and when this is so the fact will be indicated by using a subscript rather than by the usual index. Thus, for the quantity $\rho=\frac{1}{2}(\sqrt{5}-1)$ of $(7)$, when $(\sqrt{5}-2)$ occurs as a base it will be designated as $\rho_{3}$ rather than $\rho^{3}$.

\section{Functional equations}

3.1 Single variable. There are many such equations, of which the following will suffice for our needs here.

$$
\begin{gathered}
\frac{1}{2} \mathbf{L i}_{2}\left(z^{2}\right)=\mathrm{Li}_{2}(z)+\mathrm{Li}_{2}(-z) . \\
\mathrm{Li}_{2}(-z)+\mathrm{Li}_{2}(-1 / z)+\frac{1}{2} \log ^{2} z=2 \mathrm{Li}_{2}(-1)=-\pi^{2} / 6 . \\
\mathrm{Li}_{2}(z)+\mathrm{Li}_{2}(1-z)=\pi^{2} / 6-\log (z) \log (1-z) . \\
\mathrm{Li}_{2}(z)+\mathrm{Li}_{2}(-z /(1-z))=-\frac{1}{2} \log ^{2}(1-z) .
\end{gathered}
$$

All can be readily verified by differentiation. The first, known as the duplication formula, permits negative arguments to be replaced by positive ones. The second provides for an inversion of the arguments, and involves only real values if $z$ is real and positive. The constant term $2 \mathrm{Li}_{2}(-1)$ in (13) comes essentially as an integration constant by taking $z=1$. If we then take $z=-1=e^{i \pi}$ and use (12) to relate $\mathrm{Li}_{2}(-1)$ to $\mathrm{Li}_{2}(1)$ we readily get the well-known value $\mathrm{Li}_{2}(1)=\pi^{2} / 6$. Equations (13) to (15) are not all distinct-by simple re-arrangement of the variables one can deduce the last from the second two.

3.2 Two variables; algebraic form. The basic two-variable relation is usually attributed to Abel [1], though an equivalent formula was produced much earlier by Spence [13]. Various minor re-arrangements exist, due to different authors, and the following three are associated with the names of Hill [5], Kummer [6] and Schaeffer [12] respectively:

$$
\begin{aligned}
\mathrm{Li}_{2}(x y)= & \mathrm{Li}_{2}(x)+\mathrm{Li}_{2}(y)+\mathrm{Li}_{2}\left(-x \cdot \frac{1-y}{1-x}\right) \\
& +\mathrm{Li}_{2}\left(-y \cdot \frac{1-x}{1-y}\right)+\frac{1}{2} \log ^{2}\left(\frac{1-x}{1-y}\right),
\end{aligned}
$$




$$
\begin{aligned}
\mathrm{Li}_{2}\left[\frac{y(1-x)^{2}}{x(1-y)^{2}}\right]= & \operatorname{Li}_{2}\left(-y \cdot \frac{1-x}{1-y}\right)+\mathrm{Li}_{2}\left(-\frac{1}{x} \cdot \frac{1-x}{1-y}\right) \\
& +\mathrm{Li}_{2}\left(\frac{1-x}{1-y}\right)+\mathrm{Li}_{2}\left(\frac{y}{x} \cdot \frac{1-x}{1-y}\right)+\frac{1}{2} \log ^{2} x, \\
\operatorname{Li}_{2}\left(\frac{y}{x} \cdot \frac{1-x}{1-y}\right)= & \operatorname{Li}_{2}(x)-\mathrm{Li}_{2}(y)+\mathrm{Li}_{2}(y / x) \\
& +\mathrm{Li}_{2}\left(\frac{1-x}{1-y}\right)-\pi^{2} / 6+\log x \log \left(\frac{1-x}{1-y}\right) .
\end{aligned}
$$

The last two results are unsuitable for use if $x$ is negative, and a preferred form is found by inverting, through (13), those arguments becoming infinite as $x \rightarrow 0$. The resulting relations are:

$$
\begin{aligned}
\operatorname{Li}_{2}\left[\frac{x(1-y)^{2}}{y(1-x)^{2}}\right]= & \operatorname{Li}_{2}\left(-x \cdot \frac{1-y}{1-x}\right)+\operatorname{Li}_{2}\left(\frac{x}{y} \cdot \frac{1-y}{1-x}\right) \\
& -\operatorname{Li}_{2}\left(\frac{1-x}{1-y}\right)-\operatorname{Li}_{2}\left(-y \cdot \frac{1-x}{1-y}\right) \\
& +\pi^{2} / 6-\log \left(\frac{1-y}{1-x}\right) \log \left(-\frac{1}{y} \cdot \frac{1-y}{1-x}\right)
\end{aligned}
$$

$$
\begin{aligned}
\mathrm{Li}_{2}\left(\frac{x}{y} \cdot \frac{1-y}{1-x}\right)= & \mathrm{Li}_{2}(x / y)-\mathrm{Li}_{2}(x)-\mathrm{Li}_{2}(1 / y) \\
& -\mathrm{Li}_{2}\left(\frac{1-x}{1-y}\right)-\frac{1}{2} \log ^{2}\left(-\frac{1}{y} \cdot \frac{1-y}{1-x}\right)
\end{aligned}
$$

As is readily apparent, the arguments in the above are all in the form of ratios of polynomials, so the above equations can be said to be in algebraic form.

3.3 Two variables; exponent form. The algebraic forms of (16) to (20) bear little resemblance to (10), where the arguments are all in the form of a base raised to a certain power. There are several ways of achieving such a structure, however, leading to several different families of results.

3.3.1. If it is noted that all the arguments in (16) to (20) are comprised of factors $x, y$ and $(1-x) /(1-y)$ in various combinations, then we can achieve an exponent form by writing

$$
x=u^{n}, \quad y=-u^{p-q}, \quad(1-x) /(1-y)=u^{q}
$$


where $u$ is an arbitrary base, and $n, p$ and $q$ are variable exponents, which are seen to be related, on eliminating $x$ and $y$, by

$$
u^{n}+u^{p}+u^{q}=1
$$

This equation can be looked at in one of two ways: either it relates $n, p$ and $q$ for a given base; or it determines the base if the exponents are given. If the exponents are given integers, then (22) determines an algebraic quantity $u$ as a solution of a three term polynomial equation. In either case, (16) to (18) become

$$
\begin{aligned}
\operatorname{Li}_{2}\left(-u^{n+q-p}\right)= & \operatorname{Li}_{2}\left(u^{n}\right)+\operatorname{Li}_{2}\left(-u^{q-p}\right) \\
& +\operatorname{Li}_{2}\left(-u^{n-p}\right)+\operatorname{Li}_{2}\left(u^{q}\right)+\frac{1}{2} p^{2} \log ^{2} u \\
\operatorname{Li}_{2}\left(-u^{q+p-n}\right)= & \operatorname{Li}_{2}\left(u^{q}\right)+\operatorname{Li}_{2}\left(-u^{p-n}\right) \\
& +\operatorname{Li}_{2}\left(u^{p}\right)+\operatorname{Li}_{2}\left(-u^{q-n}\right)+\frac{1}{2} n^{2} \log ^{2} u \\
\operatorname{Li}_{2}\left(-u^{q-n}\right)= & \operatorname{Li}_{2}\left(u^{n}\right)-\operatorname{Li}_{2}\left(-u^{q-p}\right) \\
& +\operatorname{Li}_{2}\left(-u^{q-p-n}\right)+\operatorname{Li}_{2}\left(u^{p}\right)-\pi^{2} / 6+n p \log ^{2} u
\end{aligned}
$$

Two things may be noted about this triplet of equations:

(i) Apart from minor re-arrangements based on (13), they may be inferred one from another by interchanging $n, p$ and $q$.

(ii) Indices involving all three exponents occur once each. Indices involving two exponents occur twice. Apart from this there is no duplication unless $n, p$ and $q$ happen to have certain values such that, for instance, $n-p=q$.

Equations (23) to (25) may be said to be in exponent form.

3.3.2. A different sequence comes by taking (26)

$$
x=-v^{n}, \quad y=-v^{p-q}, \quad(1-x) /(1-y)=v^{q}
$$

where

$$
v^{p}+v^{q}-v^{n}=1, \quad n>q+p .
$$

(The inequality on $n$ is imposed to ensure a real root for $v$ in $0<v<1$.)

If these values are inserted in (16), (19) and (20) we get

$$
\begin{aligned}
\operatorname{Li}_{2}\left(v^{n+q-p}\right)= & \operatorname{Li}_{2}\left(-v^{n}\right)+\operatorname{Li}_{2}\left(-v^{q-p}\right) \\
& +\operatorname{Li}_{2}\left(v^{n-p}\right)+\operatorname{Li}_{2}\left(v^{q}\right)+\frac{1}{2} p^{2} \log ^{2} v \\
\operatorname{Li}_{2}\left(v^{n-q-p}\right)= & \operatorname{Li}_{2}\left(v^{n-p}\right)+\operatorname{Li}_{2}\left(v^{n-q}\right) \\
- & \operatorname{Li}_{2}\left(v^{p}\right)-\operatorname{Li}_{2}\left(v^{q}\right)+\pi^{2} / 6-p q \log ^{2} v \\
\operatorname{Li}_{2}\left(v^{n+p-q}\right)= & \operatorname{Li}_{2}\left(-v^{n}\right)+\operatorname{Li}_{2}\left(-v^{p-q}\right) \\
& +\operatorname{Li}_{2}\left(v^{n-q}\right)+\operatorname{Li}_{2}\left(v^{p}\right)+\frac{1}{2} q^{2} \log ^{2} v
\end{aligned}
$$


Again there are three single arguments and six pairs. However, (28) to (30) exhibit only two distinct negative arguments, whilst (23) to (25) have six. Hence, when all arguments are converted into positive ones via (12), (23) to (25) contain 15 distinct exponents whilst (28) to (30) contain 11; in each case there are but three equations among them. Thus although we have indeed produced two families of equations of the form of (11), they suffer both from paucity and, in general, sparseness.

3.3.3. The above shortcomings can be avoided somewhat if the base happens to be one for which two equations of the type (22) and/or (27) are satisfied, though with different exponents. The number of equations then doubles, though there may be some redundancy amongst them. As an example, take the case of a base $w$ satisfying

$$
w^{m}+w^{m+k}+w^{m+2 k}=1 .
$$

The series on the left can be summed to $w^{m}\left(1-w^{3 k}\right) /\left(1-w^{k}\right)$ and the equation re-written in the form

$$
w^{k}+w^{m}-w^{m+3 k}=1 .
$$

This is of the form (27), and we are led to the following six relations among 12 distinct positive arguments. However, one of these equations must be considered redundant since, with the help of the others, it is reducible to (12).

$$
\begin{aligned}
& \mathrm{Li}_{2}\left(w^{2 m+2 k}\right)=\operatorname{Li}_{2}\left(-w^{m+3 k}\right)+\operatorname{Li}_{2}\left(-w^{m-k}\right) \\
& +\mathrm{Li}_{2}\left(w^{m+2 k}\right)+\mathrm{Li}_{2}\left(w^{m}\right)+\frac{1}{2} k^{2} \log ^{2} w . \\
& \mathrm{Li}_{2}\left(w^{4 k}\right)=\mathrm{Li}_{2}\left(-w^{m+3 k}\right)+\mathrm{Li}_{2}\left(-w^{k-m}\right) \\
& +\mathrm{Li}_{2}\left(w^{3 k}\right)+\mathrm{Li}_{2}\left(w^{k}\right)+\frac{1}{2} m^{2} \log ^{2} w . \\
& \mathrm{Li}_{2}\left(w^{2 k}\right)=\mathrm{Li}_{2}\left(w^{m+2 k}\right)+\operatorname{Li}_{2}\left(w^{3 k}\right) \\
& -\operatorname{Li}_{2}\left(w^{k}\right)-\operatorname{Li}_{2}\left(w^{m}\right)+\pi^{2} / 6-m k \log ^{2} w . \\
& \operatorname{Li}_{2}\left(-w^{m+k}\right)=\operatorname{Li}_{2}\left(w^{m}\right)+\operatorname{Li}_{2}\left(w^{m+2 k}\right)-\pi^{2} / 6+\frac{1}{2} m(m+2 k) \log ^{2} w . \\
& \operatorname{Li}_{2}\left(-w^{2 k}\right)=\operatorname{Li}_{2}\left(w^{m}\right)-\operatorname{Li}_{2}\left(-w^{k}\right)+\operatorname{Li}_{2}\left(-w^{k-m}\right) \\
& +\mathrm{Li}_{2}\left(w^{m+k}\right)-\pi^{2} / 6+m(m+k) \log ^{2} w . \\
& \operatorname{Li}_{2}\left(-w^{m+3 k}\right)=\operatorname{Li}_{2}\left(w^{m+2 k}\right)+\operatorname{Li}_{2}\left(-w^{k}\right) \\
& +\mathrm{Li}_{2}\left(w^{m+k}\right)+\mathrm{Li}_{2}\left(-w^{2 k}\right)+\frac{1}{2} m^{2} \log ^{2} w .
\end{aligned}
$$

3.3.4. A different sequence comes from taking $y=-x$ in (16) to (18), and suggests the equation

$$
\left(1-U^{q}\right) /\left(1+U^{q}\right)=U^{p}, \text { or } U^{p}+U^{q}+U^{p+q}=1 .
$$


This can either be put directly into the equations, or one could use (23) to (25), for which (39) is a special case of (22). On removing the negative arguments via (12) we obtain three basic relations among nine arguments. A further (redundant) relation comes from combining the first two in a convenient way, and a fifth is an alternative way of writing the third. They are

$$
\begin{aligned}
& \mathrm{Li}_{2}\left(U^{4 q}\right)=3 \mathrm{Li}_{2}\left(U^{2 q}\right)+2 \mathrm{Li}_{2}\left(U^{p+q}\right)+2 \mathrm{Li}_{2}\left(U^{p-q}\right) \\
& -\mathrm{Li}_{2}\left(U^{2 p-2 q}\right)-\pi^{2} / 3+q(2 p-q) \log ^{2} U \\
& \mathrm{Li}_{2}\left(U^{4 p}\right)=3 \mathrm{Li}_{2}\left(U^{2 p}\right)+2 \mathrm{Li}_{2}\left(U^{p+q}\right) \\
& +\mathrm{Li}_{2}\left(U^{2 p-2 q}\right)-2 \mathrm{Li}_{2}\left(U^{p-q}\right)+q^{2} \log ^{2} U, \\
& \mathrm{Li}_{2}\left(U^{2 p}\right)=4 \mathrm{Li}_{2}\left(U^{p}\right)+4 \mathrm{Li}_{2}\left(U^{q}\right)-\mathrm{Li}_{2}\left(U^{2 q}\right)-\frac{1}{2} \pi^{2}+2 p q \log ^{2} U, \\
& \mathrm{Li}_{2}\left(U^{4 p}\right)=3 \mathrm{Li}_{2}\left(U^{2 p}\right)+4 \mathrm{Li}_{2}\left(U^{p+q}\right)-\mathrm{Li}_{2}\left(U^{4 q}\right) \\
& +3 \mathrm{Li}_{2}\left(U^{2 q}\right)-\pi^{2} / 3+2 p q \log ^{2} U \text {, } \\
& \chi_{2}\left(U^{p}\right)+\chi_{2}\left(U^{q}\right)=\pi^{2} / 8-\frac{1}{2} p q \log ^{2} U \text {. }
\end{aligned}
$$

3.3.5. Another useful sequence comes from the two-term relation

$$
V^{p}+V^{q}=1 \text {. }
$$

This can be used directly in the algebraic form of the equations, or it can be converted to a form of (22) by writing it in one of the two alternative forms

$$
\begin{aligned}
& \text { (a) } V^{p}+V^{p+q}+V^{2 q}=1, \\
& \text { (b) } V^{q}+V^{p+q}+V^{2 p}=1 .
\end{aligned}
$$

This leads to six equations, of which three can be reduced to forms of (13) to (15); and, remembering that there is an inter-relation among these, we are left with five independent forms which can be conveniently chosen as

$$
\begin{aligned}
& \mathrm{Li}_{2}\left(V^{p}\right)+\mathrm{Li}_{2}\left(V^{q}\right)=\pi^{2} / 6-p q \log ^{2} V, \\
& \mathrm{Li}_{2}\left(V^{q}\right)+\mathrm{Li}_{2}\left(-V^{q-p}\right)=-\frac{1}{2} p^{2} \log ^{2} V, \\
& \operatorname{Li}_{2}\left(V^{p+q}\right)=\mathrm{Li}_{2}\left(-V^{2 q-p}\right)-\mathrm{Li}_{2}\left(-V^{q-2 p}\right)-\left(3 p^{2} / 2\right) \log ^{2} V \text {, } \\
& \mathrm{Li}_{2}\left(V^{3 q-3 p}\right)=\mathrm{Li}_{2}\left(V^{2 q-2 p}\right)+\mathrm{Li}_{2}\left(V^{q-p}\right) \\
& +\mathrm{Li}_{2}\left(-V^{2 q-p}\right)+\mathrm{Li}_{2}\left(-V^{q-2 p}\right)+\frac{1}{2} p^{2} \log ^{2} V, \\
& \mathrm{Li}_{2}\left(-V^{3 q}\right)=\mathrm{Li}_{2}\left(V^{2 q}\right)+\mathrm{Li}_{2}\left(-V^{q}\right) \\
& +\mathrm{Li}_{2}\left(V^{q+p}\right)+\mathrm{Li}_{2}\left(-V^{2 q-p}\right)+\frac{1}{2} p^{2} \log ^{2} V .
\end{aligned}
$$

Special cases of interest come from taking $q=1$ or $p-1$. 
If $V$ satisfies more than one equation of the form (45), the number of equivalent three-term equations proliferates rapidly, producing many more relations of the form (47) to (51), though with considerable redundancies. But there appears to be only one such base with all the exponents integral, and this is discussed in detail in Section 5.17.

3.3.6. There is an instructive alternative form of writing equations such as (29). To see how this comes about, consider first how it would apply to the simple logarithm, which in algebraic form satisfies $\log x+\log y=\log (x y)$. If we take $x=W^{m}, y=W^{n}, x y=W^{p}$, then clearly $W^{m} \cdot W^{n}=W^{p}$, or $p=m+n$, determines $p$ in terms of $m$ and $n$. The exponent form of the equation is $\log W^{m}+$ $\log W^{n}=\log W^{p}$, and if we define $f(m)=\log W^{m}$ the equation reduces to

$$
f(m)+f(n)=f(p)
$$

(essentially the law of addition of indices).

The corresponding form of (29), with $\mathrm{Li}_{2}\left(v^{p}\right)$ written as $g(p)$, is

(53) $g(n-q-p)=g(n-p)+g(n-q)-g(p)-g(q)+\pi^{2} / 6-p q \log ^{2} v$

with $n, p$ and $q$ related by (27). This is a much more "readable" equation than (19), from which it was derived, and suggests that it may be of value to emphasize more the exponent form of the equations. However, the simplicity of (53) is somewhat misleading, because of the involved inter-relationshiop between $n, p, q$ and $v$.

The "linear-looking" form of (53) is strongly reminiscent of the functional equation for Clausen's function [3], the imaginary part of $\mathrm{Li}_{2}\left(e^{i a}\right)$, for which linear combinations of angles occur in an addition-type formula. This lead is taken up in Section 6, where it is shown to yield a new equation in exponent form with 15 dilogarithmic terms. The defining equation for the base contains 11 terms, and considerably expands the range of equations from the rather limited forms of the present section. Further extensions are possible, in principle, by the same method.

\section{Relations in the complex plane}

It might be thought that all possible relations could be ultimately deduced from Abel's equation or one of its variants. Although this may be true, and Watson's success with $2 \cos (3 \pi / 7)$ by this method notwithstanding, it seems to be the case that when the base is a trigonometric function of a submultiple of $\pi$ there are results that are much more readily obtainable from a consideration of $\operatorname{Li}_{2}(z)$ in the complex plane. 
Although these trigonometric terms are all solutions of rational algebraic equations, there is a distinguishing feature that appears to be significant, and which divides them from solutions of such equations as (22) or (45). The distinction is clearly evident in the case of the cubic equation. For example, (45) with $p=1, q=3$ has a single real root, expressible in terms of real radicals. But the cubic equation giving rise to $2 \cos (3 \pi / 7)$ also has two other real roots, expressible in simple trigonometric form, and none of these can be evaluated in terms of real radicals. Thus we find that the solutions to the base equations divide up naturally into two major groups distinguished by whether or not they are expressible in a fairly natural way in simple trigonometric form. For convenience we can label these groups of solutions as "trigonometric" and "algebraic", even though the trigonometric values are, of course, all algebraic numbers.

These two groups have a certain amount of overlap at the more "primitive" end, where solutions of linear or quadratic equations are involved. For example, $\rho=2 \cos (2 \pi / 5)$ satisfies $\rho^{2}+\rho=1$, and most of Coxeter's formulae (10) are obtainable via (47) to (51): but (10d) is apparently inaccessible by this route. Again, the value $1 / \sqrt{3}$ can somewhat arbitrarily be identified with $\tan (\pi / 6)$, and although some of its properties can be found via Abel's equations, others apparently cannot. They are, however, obtainable from formulae involving the dilogarithm of tangents in the complex plane, thus confirming the relevance of the identification in this case.

4.1 Functional equations in the complex plane. Of the numerous equations extant, we quote the following, all taken, with minor changes, from Chapter 5 of reference 9, where the notation $\mathrm{Li}_{2}(x, a)$ is used for the real part of $\mathrm{Li}_{2}\left(x e^{i a}\right)$.

$$
\begin{aligned}
\mathrm{Li}_{2}\left(\tan a, \frac{1}{2} \pi-2 a\right) & =a^{2}+\frac{3}{4} \mathrm{Li}_{2}\left(\tan ^{2} a\right)-\frac{1}{8} \mathrm{Li}_{2}\left(\tan ^{4} a\right) . \\
\mathrm{Li}_{2}(x, \pi / 3) & =\frac{1}{6} \mathrm{Li}_{2}\left(-x^{3}\right)-\frac{1}{2} \mathrm{Li}_{2}(-x) . \\
\mathrm{Li}_{2}(x, \pi / 4)= & \frac{1}{4} \mathrm{Li}_{2}\left(x \sqrt{2}-x^{2}\right) \\
& -\frac{1}{2} \mathrm{Li}_{2}\left(\frac{-x}{\sqrt{2}-x}\right)+\frac{1}{8} \mathrm{Li}_{2}\left(\frac{-x^{2}}{(\sqrt{2}-x)^{2}}\right) . \\
\mathrm{Li}_{2}(x, \pi / 6)= & \frac{1}{12} \operatorname{Li}_{2}\left(-x^{2}\right)-\frac{1}{4} \operatorname{Li}_{2}(-x) \\
& -\frac{1}{2} \operatorname{Li}_{2}\left(\frac{-x}{\sqrt{3}-x}\right)+\frac{1}{4} \mathrm{Li}_{2}\left(x \sqrt{3}-x^{2}\right) .
\end{aligned}
$$




$$
\begin{aligned}
\operatorname{Li}_{2}\left[-\frac{\sin (M-1) a}{\sin a}, M a\right]= & \frac{1}{2} a^{2} M(M-1)-\frac{1}{2} \sum_{1}^{M-1} \operatorname{Li}_{2}\left(\frac{\sin ^{2} a}{\sin ^{2} r a}\right) \\
& +\sum_{2}^{M-1} \log \left(\frac{\sin r a}{\sin a}\right) \log \left[\frac{\sin (r-1) a}{\sin r a}\right] .
\end{aligned}
$$

The special case $a=\pi / M$ of $(58)$ is

$$
\begin{aligned}
\sum_{2}^{M-1} \operatorname{Li}_{2}\left[\frac{\sin ^{2}(\pi / M)}{\sin ^{2}(r \pi / M)}\right] & =\frac{M-2}{2 M} \pi^{2} \\
& +2 \sum_{2}^{M-1} \log \left[\frac{\sin (r \pi / M)}{\sin (\pi / M)}\right] \log \left[\frac{\sin ((r-1) \pi / M)}{\sin (r \pi / M)}\right]
\end{aligned}
$$

4.2 A formula of Richmond and Szekeres. A formula, which was at first believed to be of a quite different character from (59), was found by Richmond and Szekeres [10] by evaluating the coefficients of expansiion of certain RogersRamanujan partition identities (due to George Andrews). Defining

$$
\text { (a) } \quad d_{1}=2\left(1-\cos \frac{\pi}{2 r+3}\right)
$$

(b) $\quad d_{j+1}=d_{j} \prod_{i=1}^{j}\left(1-d_{i}\right)^{-2}, \quad j=1,2, \ldots, r-1$,

the relation is (in dilogarithmic form)

$$
\sum_{i=1}^{r} \operatorname{Li}_{2}\left(d_{i}\right)=\frac{\pi^{2}}{3} \frac{r}{2 r+3}-\frac{1}{2} \sum_{i=1}^{r} \log d_{i} \log \left(1-d_{i}\right) \text {. }
$$

If we take $r \rightarrow \infty$ the formula goes into

$$
\sum_{k=2}^{\infty} \operatorname{Li}_{2}\left(1 / k^{2}\right)=\pi^{2} / 6+\sum_{2}^{\infty} \log k \log \left(1-1 / k^{2}\right)
$$

which Szekeres has put in terms of the Riemann zeta-function,

$$
\sum_{k=1}^{\infty}\left[\frac{1}{k^{2}} \zeta(2 k)-\frac{1}{k} \zeta^{\prime}(2 k)\right]=\frac{\pi^{2}}{3} \text {. }
$$

Equation (62) can be found from (58) by taking the limit $a \rightarrow 0$. Writing $M+1$ for $M$ gives

$$
\mathrm{Li}_{2}(-M)=\frac{1}{2} \sum_{1}^{M} \mathrm{Li}_{2}\left(1 / k^{2}\right)+\sum_{2}^{M} \log k \log \left(\frac{k-1}{k}\right)
$$

from which (63) can be deduced by taking the limit $M \rightarrow \infty$ and using (13). Equation (64) can also be obtained directly via Abel's equation. 
The similarity between (62) and (64) suggests that, after all, (59) and (61) may be related, and a brief outline of a proof follows.

Writing $d_{j}=1 / D_{j}^{2}$ in $(60 \mathrm{~b})$, inverting and taking the square root gives

$$
\begin{aligned}
& \text { (a) } D_{j+1}=D_{j} \prod_{i=1}^{j}\left(1-1 / D_{i}^{2}\right), \\
& \text { (b) } D_{j+2}=D_{j+1} \prod_{i=1}^{j+1}\left(1-1 / D_{i}^{2}\right)
\end{aligned}
$$

where $(65 \mathrm{~b})$ comes by putting $j+1$ for $j$ in (65a).

Dividing the two equations gives

$$
D_{j} D_{j+2}=D_{j+1}^{2}-1 \text {. }
$$

Recalling the identity $\sin ^{2} x-\sin ^{2} y=\sin (x+y) \sin (x-y)$ enables (66) to be solved by inspection in the form $D_{j}^{-1}=\sin B / \sin (j B+C)$ with $B$ and $C$ constants to be determined by $(60)$. Hence it is found that

$$
d_{j}=\sin ^{2}\left(\frac{\pi}{2 r+3}\right) / \sin ^{2}\left(\pi \frac{r+2-j}{2 r+3}\right)
$$

and (61) goes over into (59) with $M=2 r+3$.

One of Watson's results, (76a), can be found from (59) with $M=7$. It is not known if the other two can also be deduced from this equation.

\section{Numerical results}

Greek lower case letters $\alpha$ to $\omega$ (except $\pi$ ) will be used to designate specific numerical bases, though in no particular order. Watson's notation $\alpha, \beta, \gamma$ has been retained, and, as used earlier, $\rho$ is $\frac{1}{2}(\sqrt{5}-1)$. A power of a base, when itself a base, is indicated by a subscript. Many of the quantities encountered satisfy equations like (22), (27), (45), or several of them together. All of the "algebraic" bases, and some of the trigonometric bases have results given by the equations of Sections 3.3 or 6 . The details of the derivations, which equations are used, and so on, are not given unless some particular point of interest is involved. In general, most of the results are straightforward, given the material of Sections 3, 4 and 6.

$5.1 \rho=\frac{1}{2}(\sqrt{5}-1)=2 \cos (2 \pi / 5)$; Landen's and Coxeter's formulae. The equations satisfied by $\rho$ are

$$
\begin{aligned}
& \rho^{2}+\rho=1, \quad \rho+\rho^{3}+\rho^{4}=1, \quad 2 \rho^{2}++\rho^{3}=1, \\
& (1-\rho) /(1+\rho)=\rho^{3}, \quad 2 \rho-\rho^{3}=1 .
\end{aligned}
$$


It lies at the confluence of (39) $(q=1, p=3)$ and (45) $(p=1, q=2)$ and is at the same time both of the special cases $p=1$ and $p=q-1$. All the known results are comprised in (8), (9) and (10), but they can be re-written in the following instructive way:

5.1.1. Landen's and Coxeter's results.

(a) $\mathrm{Li}_{2}(\rho)+\mathrm{Li}_{2}\left(\rho^{2}\right)=\pi^{2} / 6-2 \log ^{2} \rho$,

(b) $\mathrm{Li}_{2}(\rho)-\mathrm{Li}_{2}\left(\rho^{2}\right)=\pi^{2} / 30$,

(c) $\operatorname{Li}_{2}\left(\rho^{6}\right)=4 \mathrm{Li}_{2}\left(\rho^{3}\right)+3 \mathrm{Li}_{2}\left(\rho^{2}\right)-6 \operatorname{Li}_{2}(\rho)+7 \pi^{2} / 30$,

(d) $\operatorname{Li}_{2}\left(\rho^{12}\right)=2 \operatorname{Li}_{2}\left(\rho^{6}\right)+3 \operatorname{Li}_{2}\left(\rho^{4}\right)+4 \operatorname{Li}_{2}\left(\rho^{3}\right)$ $-6 \operatorname{Li}_{2}\left(\rho^{2}\right)+\pi^{2} / 10$,

(e) $\operatorname{Li}_{2}\left(\rho^{20}\right)=2 \operatorname{Li}_{2}\left(\rho^{10}\right)+15 \mathrm{Li}_{2}\left(\rho^{4}\right)-10 \mathrm{Li}_{2}\left(\rho^{2}\right)+\pi^{2} / 5$,

(f) $\chi_{2}(\rho)=\pi^{2} / 12-\frac{3}{4} \log ^{2} \rho$,

(g) $\chi_{2}\left(\rho^{3}\right)=\pi^{2} / 24-\frac{3}{4} \log ^{2} \rho$.

5.1.2. The base $\rho_{2}=\rho^{2}=\frac{1}{2}(3-\sqrt{5})=2(1-\cos \pi / 5)$ satisfies the equation

$$
3 \rho_{2}-\rho_{2}^{2}=1 \text {. }
$$

Results are taken from (69) and are

(a) $\operatorname{Li}_{2}\left(\rho_{2}\right)=\pi^{2} / 15-\frac{1}{4} \log ^{2} \rho_{2}$,

(b) $\operatorname{Li}_{2}\left(\rho_{2}^{6}\right)=3 \mathrm{Li}_{2}\left(\rho_{2}^{3}\right)+3 \mathrm{Li}_{2}\left(\rho_{2}^{2}\right)-3 \mathrm{Li}_{2}\left(\rho_{2}\right)+\pi^{2} / 15$,

(c) $\operatorname{Li}_{2}\left(\rho_{2}^{10}\right)=2 \mathrm{Li}_{2}\left(\rho_{2}^{5}\right)+15 \mathrm{Li}_{2}\left(\rho_{2}^{2}\right)-10 \mathrm{Li}_{2}\left(\rho_{2}\right)+\pi^{2} / 5$.

5.1.3. The base $\rho_{3}=\rho^{3}=\sqrt{5}-2=\tan (\pi / 30) \cot (2 \pi / 15)$ satisfies

$$
\rho_{2}^{2}+4 \rho_{3}=1 \text {. }
$$

Results are taken from (69) and are

(a) $\operatorname{Li}_{2}\left(\rho_{3}^{2}\right)=4 \mathrm{Li}_{2}\left(\rho_{3}\right)-\pi^{2} / 6+\frac{1}{3} \log ^{2} \rho_{3}$,

(b) $\chi_{2}\left(\rho_{3}\right)=\pi^{2} / 24-\frac{1}{12} \log ^{2} \rho_{3}$.

$5.2 \alpha, \beta, \gamma ;$ Watson's formulae. Watson [14] examined solutions of the equation $x^{3}+2 x^{2}-x-1=0$, which has one positive root and two negative roots. Denoting them by $\alpha,-\beta,-1 / \gamma$ respectively, then all three quantities $\alpha, \beta$ and $\gamma$ lie 
between 0 and 1 and satisfy
(a) $\alpha^{3}+2 \alpha^{2}-\alpha-1=0$,
(b) $\beta^{3}-2 \beta^{2}-\beta+1=0$,
(c) $\gamma^{3}-\gamma^{2}-2 \gamma+1=0$.

They have the values
(a) $\alpha=\frac{1}{2} \sec (2 \pi / 7)$,
(b) $\beta=\frac{1}{2} \sec (\pi / 7)=1 /(1+\alpha)$,
(c) $\gamma=2 \cos (3 \pi / 7)=\alpha /(1+\alpha)$.

Equation (74a) can be put in a number of different forms; for example $1-\alpha=\alpha^{2} /(1+\alpha)^{2}$. Using (12) to (15) a number of times over he deduced a relation in which $\operatorname{Li}_{2}(\alpha)$ and $\mathrm{Li}_{2}\left(\alpha^{2}\right)$ are all accumulated seven times, whilst the quantity they equal involves $\pi^{2} / 6$. The final relation, together with corresponding relations for $\beta$ and $\gamma$, similarly found, is
(a) $\mathrm{Li}_{2}(\alpha)-\mathrm{Li}_{2}\left(\alpha^{2}\right)=\pi^{2} / 42+\log ^{2} \alpha$
(b) $2 \operatorname{Li}_{2}(\beta)+\mathrm{Li}_{2}\left(\beta^{2}\right)=5 \pi^{2} / 21-2 \log ^{2} \beta$
(c) $2 \mathrm{Li}_{2}(\gamma)+\mathrm{Li}_{2}\left(\gamma^{2}\right)=4 \pi^{2} / 21-\log ^{2} \gamma$.

For details of the derivation the reader is referred to Watson's orginal paper, or to the briefer discussion in reference 9 .

$5.3 \kappa, \lambda, \mu ;$ Loxton's formula and extrapolations therefrom. I am indebted to G. Szekeres for drawing my attention to a result of John Loxton's, obtained from a partition identity of Slater's. Using Roger's $L$-function, and defining $X=$ $2 \cos (2 \pi / 9)-1$, which is a solution of $X^{3}+3 X^{2}=1$, it can be written as

$$
L(1-X)+L\left(1-X^{2}\right)-\frac{1}{3} L\left(1-X^{3}\right)=4 \pi^{2} / 27 .
$$

It can easily be reduced to a form strikingly similar to Watson's (76a), suggesting that two further such relations may also exist. The likely form of these is indicated by the factoring considerations discussed later in Section 7.4, and is completely determined apart from a simple rational multiple of $\pi^{2} / 54$. The presumptive relations were set up and then verified numerically. Defining

$$
\begin{aligned}
& \text { (a) } \kappa=\frac{1}{2} \sec (\pi / 9), \\
& \text { (b) } \lambda=\frac{1}{2} \sec (2 \pi / 9)=1 /(1+\kappa), \\
& \text { (c) } \mu=2 \cos (4 \pi / 9)=\kappa(1+\kappa),
\end{aligned}
$$


which satisfy
(a) $\kappa^{3}+3 \kappa^{2}-1=0$,
(b) $\lambda^{3}-3 \lambda^{2}+1=0$,
(c) $\mu^{3}-3 \mu+1=0$,

the following relations were found to exist:
(a) $\operatorname{Li}_{2}(\kappa)+\operatorname{Li}_{2}\left(\kappa^{2}\right)-\frac{1}{3} \operatorname{Li}_{2}\left(\kappa^{3}\right)=7 \pi^{2} / 54-\log ^{2} \kappa$,
(b) $\operatorname{Li}_{2}(-\lambda)+\operatorname{Li}_{2}\left(\lambda^{2}\right)-\frac{1}{3} \operatorname{Li}_{2}\left(-\lambda^{3}\right)=\pi^{2} / 54-\log ^{2} \lambda$,
(c) $\mathrm{Li}_{2}(-\mu)+\mathrm{Li}_{2}\left(\mu^{2}\right)-\frac{1}{3} \mathrm{Li}_{2}\left(-\mu^{3}\right)=-\pi^{2} / 54$.

They can also be put in the form

(a) $\mathrm{Li}_{2}\left(\kappa^{3}\right)=3 \mathrm{Li}_{2}\left(\kappa^{2}\right)+3 \mathrm{Li}_{2}(\kappa)-7 \pi^{2} / 18+3 \log ^{2} \kappa$,

(b) $\operatorname{Li}_{2}\left(\lambda^{6}\right)=2 \operatorname{Li}_{2}\left(\lambda^{3}\right)+9 \operatorname{Li}_{2}\left(\lambda^{2}\right)-6 \operatorname{Li}_{2}(\lambda)-\pi^{2} / 9+6 \log ^{2} \lambda$,

(c) $\mathrm{Li}_{2}\left(\mu^{6}\right)=2 \mathrm{Li}_{2}\left(\mu^{3}\right)+9 \mathrm{Li}_{2}\left(\mu^{2}\right)-6 \mathrm{Li}_{2}(\mu)+\pi^{2} / 9$.

Equations (80a) or (81a) are, of course, Loxton's (77) in slightly different guise. The remaining pair are new. They have been verified numerically but at time of writing lack any analytic derivation. Attempts to derive them from Abel's equation, from (59) with $M=9$, or from (80a), have not so far been successful.

$5.4 \varepsilon=1 / \sqrt{3}=\tan (\pi / 6)=\frac{1}{2} \sec (\pi / 6)$.

5.4.1. For use with (22), $\varepsilon$ satisfies

$$
\varepsilon^{2}+\varepsilon^{2}+\varepsilon^{2}=1
$$

and yields but a single result, best expressed in terms of $\varepsilon_{2}$ where

$$
\begin{gathered}
\varepsilon_{2}=\varepsilon^{2}=\frac{1}{3}, \\
6 \mathrm{Li}_{2}\left(\varepsilon_{2}\right)-\mathrm{Li}_{2}\left(\varepsilon_{2}^{2}\right)=\pi^{2} / 3-\log ^{2} \varepsilon_{2} .
\end{gathered}
$$

5.4.2. If (84) were an isolated result there would be no need to consider $\varepsilon$ separately from $\varepsilon_{2}$. However, there are further formulae, the simplest coming from (54) with $a=\pi / 6$, together with (57) with $x=\tan (\pi / 6)$. Using (84) to eliminate terms in $\varepsilon^{4}$ results in

$$
\begin{aligned}
\operatorname{Li}_{2}\left(\varepsilon^{6}\right)= & 2 \operatorname{Li}_{2}\left(\varepsilon^{3}\right)-3 \operatorname{Li}_{2}\left(\varepsilon^{2}\right)-6 \operatorname{Li}_{2}(\varepsilon) \\
& +2 \pi^{2} / 3-3 \log ^{2}\left(1-\varepsilon^{2}\right)-3 \log ^{2}\left(\frac{1-\varepsilon^{2}}{\varepsilon^{2}}\right) .
\end{aligned}
$$


This is the only such relation studied here for which the logarithmic terms do not reduce to a simple multiple of the square of the logarithm of the base. The form (85) is not in any simpler if the $L$-function is used instead of $\mathrm{Li}_{2}$.

$5.5 \delta=\frac{1}{2}=\sin (\pi / 6)$ or $\sin ^{2}(\pi / 4) ; \delta_{1 / 2}=\delta^{1 / 2}=\sin (\pi / 4)$. The equation satisfied, for use with (22), is

$$
\delta+\delta^{2}+\delta^{2}=1
$$

and yields only two results:

$$
\operatorname{Li}_{2}(\delta)=\pi^{2} / 12-\frac{1}{2} \log ^{2} \delta \quad \text { (Euler) }
$$

and

$$
\mathrm{Li}_{2}\left(\delta^{6}\right)=2 \mathrm{Li}_{2}\left(\delta^{3}\right)+6 \mathrm{Li}_{2}\left(\delta^{2}\right)-6 \mathrm{Li}_{2}(\delta)+\pi^{2} / 6
$$

$5.6 \chi=\tan (\pi / 12)=2-\sqrt{3}$. The equation is

$$
4 x-x^{2}=1
$$

but this cannot be used directly in (22). Along the lines of (39) one can take

$$
(1-U) /(1+U)=i U
$$

from which $U=e^{-i \pi / 4}(\sqrt{3}-1) / \sqrt{2}$, and the arguments involve $i U^{2}=\chi$. The imaginary part of the equation involves the inverse-tangent integral and gives a result already known from other sources; the real part gives

$$
\mathrm{Li}_{2}\left(\chi^{4}\right)=2 \mathrm{Li}_{2}\left(\chi^{2}\right)+16 \mathrm{Li}_{2}(\chi)+2 \log ^{2} \chi-5 \pi^{2} / 6 .
$$

A further result comes from (54) and (55) with $a=\pi / 6, x=\tan (\pi / 12)$ and can be written, after combination with (91),

$$
\mathrm{Li}_{2}\left(\chi^{6}\right)=2 \mathrm{Li}_{2}\left(\chi^{3}\right)+9 \mathrm{Li}_{2}\left(\chi^{2}\right)-30 \mathrm{Li}_{2}(\chi)-3 \log ^{2} \chi+4 \pi^{2} / 3 .
$$

It does not seem possible to obtain this result directly from Abel's equation.

$5.7 \tau=\sqrt{2}-1=\tan (\pi / 8)$.

5.7.1. The equation for use in (22) is

$$
\tau+\tau+\tau^{2}=1
$$

or, alternatively, $(1-\tau) /(1+\tau)=\tau$ in (40) to (44). Only two relations survive and can be written
(a) $\operatorname{Li}_{2}\left(\tau^{2}\right)=4 \mathrm{Li}_{2}(\tau)-\pi^{2} / 4+\log ^{2} \tau$ or
(b) $\chi_{2}(\tau)=\pi^{2} / 16-\frac{1}{4} \log ^{2} \tau$ 
and

(a) $\mathrm{Li}_{2}\left(\tau^{4}\right)=6 \mathrm{Li}_{2}\left(\tau^{2}\right)-4 \mathrm{Li}_{2}(\tau)+\pi^{2} / 12$ or

(b) $\mathrm{Li}_{2}\left(\tau^{4}\right)=4 \mathrm{Li}_{2}\left(\tau^{2}\right)+4 \mathrm{Li}_{2}(\tau)+2 \log ^{2} \tau-5 \pi^{2} / 12$.

5.7.2. There is one result obtainable from (94) and (95) for

$$
\tau_{2}=\tau^{2}=3-2 \sqrt{2}=\tan ^{2}(\pi / 8), \quad 6 \tau_{2}-\tau_{2}^{2}=1 .
$$

It can be written

$$
\operatorname{Li}_{2}\left(\tau_{2}^{2}\right)=5 \operatorname{Li}_{2}\left(\tau_{2}\right)-\pi^{2} / 6+\frac{1}{4} \log ^{2} \tau_{2} .
$$

5.8 Composite results for $\delta, \varepsilon, \chi$ and $\tau$. There are a number of interesting relations interconnecting dilogarithms of the preceding four bases. Composite relations of this character are not the object of the present study, but the following results seem worthy of report in the present context. They are all readily obtainable from the formulae of Section 3.
(a) $\operatorname{Li}_{2}\left(\delta^{2}\right)+2 \operatorname{Li}_{2}\left(\varepsilon^{2}\right)=\pi^{2} / 6-\left[\log ^{2} \delta+\log ^{2}\left(\delta / \varepsilon^{2}\right)\right]$,
(b) $\operatorname{Li}_{2}\left(\delta_{1 / 2}\right)-\mathrm{Li}_{2}(\tau)=\pi^{2} / 24-\frac{1}{2} \log \tau \log \left(\tau \delta^{1 / 2}\right)$,
(c) $4 \mathrm{Li}_{2}(\chi)-\mathrm{Li}_{2}\left(\chi^{2}\right)+4 \mathrm{Li}_{2}(\varepsilon)-\mathrm{Li}_{2}\left(\varepsilon^{2}\right)=\pi^{2} / 2-2 \log \varepsilon \log \chi$.

5.9 o.

5.9.1. The equations, for use in (39) and (22), are

(99) $(1-\sigma) /(1+\sigma)=\sigma^{2}, \quad \sigma+\sigma^{2}+\sigma^{3}=1, \quad \sigma+\sigma-\sigma^{4}=1$.

There are three independent equations:

(a) $2 \operatorname{Li}_{2}\left(\sigma^{3}\right)=\mathrm{Li}_{2}\left(\sigma^{2}\right)+2 \mathrm{Li}_{2}(\sigma)-\pi^{2} / 6+\log ^{2} \sigma$

(b) $\operatorname{Li}_{2}\left(\sigma^{4}\right)=3 \operatorname{Li}_{2}\left(\sigma^{2}\right)+4 \operatorname{Li}_{2}(\sigma)-\pi^{2} / 2+4 \log ^{2} \sigma$,

(c) $\mathrm{Li}_{2}\left(\sigma^{8}\right)=4 \mathrm{Li}_{2}\left(\sigma^{4}\right)-\mathrm{Li}_{2}\left(\sigma^{2}\right)-4 \mathrm{Li}_{2}(\sigma)+\pi^{2} / 3-2 \log ^{2} \sigma$.

5.9.2. $\sigma_{2}=\sigma^{2}$ satisfies

$$
\sigma_{2}^{3}+\sigma_{2}^{2}+3 \sigma_{2}=1
$$

From (100c) we get

$$
\mathrm{Li}_{2}\left(\sigma_{2}^{4}\right)=3 \mathrm{Li}_{2}\left(\sigma_{2}^{2}\right)+2 \mathrm{Li}_{2}\left(\sigma_{2}\right)-\pi^{2} / 6+\frac{1}{2} \log ^{2} \sigma_{2} .
$$

$5.10 \nu$. The equation satisfied by $\nu$ is

$$
(1-\nu) /(1+\nu)=\nu^{4}, \quad \nu+\nu^{4}+\nu^{5}=1 .
$$


(The cubic form $(1-\rho) /(1+\rho)=\rho^{3}$ was considered in Section 4.1.) The results are

(a) $\operatorname{Li}_{2}\left(\nu^{6}\right)=2 \operatorname{Li}_{2}\left(\nu^{5}\right)-\mathrm{Li}_{2}\left(\nu^{4}\right)+2 \operatorname{Li}_{2}\left(\nu^{3}\right)$

$$
+3 \operatorname{Li}_{2}\left(\nu^{2}\right)-\pi^{2} / 3+7 \log ^{2} \nu,
$$

(104) (b) $\mathrm{Li}_{2}\left(\nu^{8}\right)=4 \mathrm{Li}_{2}\left(\nu^{4}\right)-\mathrm{Li}_{2}\left(\nu^{2}\right)+4 \mathrm{Li}_{2}(\nu)-\pi^{2} / 2+8 \log ^{2} \nu$,

(c) $\mathrm{Li}_{2}\left(\nu^{16}\right)=4 \mathrm{Li}_{2}\left(\nu^{8}\right)+4 \mathrm{Li}_{2}\left(\nu^{5}\right)-5 \mathrm{Li}_{2}\left(\nu^{4}\right)$

$$
+4 \operatorname{Li}_{2}\left(\nu^{2}\right)-4 \operatorname{Li}_{2}(\nu)+\pi^{2} / 6 \text {. }
$$

$5.11 \zeta=\left(\frac{1}{2}(\sqrt{59 / 27}+1)\right)^{1 / 3}-\left(\frac{1}{2}(\sqrt{59 / 27}-1)\right)^{1 / 3}$. The equation is

$$
\zeta+\zeta+\zeta^{3}=1
$$

with results

(a) $\operatorname{Li}_{2}\left(\zeta^{4}\right)=\frac{5}{2} \operatorname{Li}_{2}\left(\zeta^{2}\right)+\mathrm{Li}_{2}(\zeta)-\pi^{2} / 6+\log ^{2} \zeta$,

(b) $\quad \mathrm{Li}_{2}\left(\zeta^{6}\right)=4 \mathrm{Li}_{2}\left(\zeta^{3}\right)+\frac{1}{2} \mathrm{Li}_{2}\left(\zeta^{2}\right)+3 \mathrm{Li}_{2}(\zeta)-\pi^{2} / 3+2 \log ^{2} \zeta$

or, in a form not involving any logarithmic terms at all,

(c) $2 \mathrm{Li}_{2}\left(\zeta^{6}\right)+9 \mathrm{Li}_{2}\left(\zeta^{2}\right)=8 \mathrm{Li}_{2}\left(\zeta^{3}\right)+4 \mathrm{Li}_{2}\left(\zeta^{4}\right)+2 \mathrm{Li}_{2}(\zeta)$

$5.12 \eta=\left(\frac{1}{4}(\sqrt{29 / 27}+1)\right)^{1 / 3}-\left(\frac{1}{4}(\sqrt{29 / 27}-1)\right)^{1 / 3}$. The equation is

$$
\eta+\eta^{3}+\eta^{3}=1
$$

with two net results

$$
\begin{aligned}
\text { (a) } \mathrm{Li}_{2}\left(\eta^{4}\right)= & 2 \mathrm{Li}_{2}\left(\eta^{3}\right)+\mathrm{Li}_{2}\left(\eta^{2}\right)+4 \mathrm{Li}_{2}(\eta)-\pi^{2} / 2+5 \log ^{2} \eta, \\
\text { (b) } \mathrm{Li}_{2}\left(\eta^{10}\right)= & 2 \mathrm{Li}_{2}\left(\eta^{5}\right)+2 \mathrm{Li}_{2}\left(\eta^{4}\right) \\
& +4 \mathrm{Li}_{2}\left(\eta^{3}\right)-4 \mathrm{Li}_{2}\left(\eta^{2}\right)+\log ^{2} \eta .
\end{aligned}
$$

$5.13 \theta=\left(\frac{1}{2}(\sqrt{31 / 27}+1)\right)^{1 / 3}-\left(\frac{1}{2}(\sqrt{31 / 27}-1)\right)^{1 / 3}$. This base is defined by $1-\theta=\theta^{3}$ and possesses four three-term equations.

$$
\begin{array}{ll}
\theta^{2}+\theta^{3}+\theta^{4}=1, & \theta+\theta^{4}+\theta^{6}=1, \\
\theta^{2}+\theta^{2}+\theta^{7}=1, & \theta+\theta^{2}-\theta^{5}=1 .
\end{array}
$$


There are some redundant relations, but the following six independent equations are readily found:

(110)

(a) $\mathrm{Li}_{2}(\theta)+\mathrm{Li}_{2}\left(\theta^{3}\right)=\pi^{2} / 6-3 \log ^{2} \theta$,

(b) $\mathrm{Li}_{2}\left(\theta^{4}\right)=2 \mathrm{Li}_{2}\left(\theta^{2}\right)+2 \mathrm{Li}_{2}(\theta)-\pi^{2} / 3+5 \log ^{2} \theta$,

(c) $\mathrm{Li}_{2}\left(\theta^{6}\right)=2 \mathrm{Li}_{2}\left(\theta^{3}\right)+6 \mathrm{Li}_{2}\left(\theta^{2}\right)+4 \mathrm{Li}_{2}(\theta)-\pi^{2}+18 \log ^{2} \theta$,

(d) $\mathrm{Li}_{2}\left(\theta^{10}\right)=2 \mathrm{Li}_{2}\left(\theta^{5}\right)+5 \mathrm{Li}_{2}\left(\theta^{2}\right)+2 \mathrm{Li}_{2}(\theta)-2 \pi^{2} / 3+13 \log ^{2} \theta$,

(e) $\mathrm{Li}_{2}\left(\theta^{14}\right)=4 \mathrm{Li}_{2}\left(\theta^{7}\right)+7 \mathrm{Li}_{2}\left(\theta^{2}\right)+2 \mathrm{Li}_{2}(\theta)-5 \pi^{2} / 6+17 \log ^{2} \theta$,

(f) $\mathrm{Li}_{2}\left(\theta^{18}\right)=2 \mathrm{Li}_{2}\left(\theta^{9}\right)+3 \mathrm{Li}_{2}\left(\theta^{6}\right)+6 \mathrm{Li}_{2}\left(\theta^{3}\right)$

$$
+9 \mathrm{Li}_{2}\left(\theta^{2}\right)+14 \mathrm{Li}_{2}(\theta)-8 \pi^{2} / 3+48 \log ^{2} \theta
$$

or, in a form free from all logarithmic terms

(g) $\quad \mathrm{Li}_{2}\left(\theta^{18}\right)+10 \mathrm{Li}_{2}\left(\theta^{3}\right)+2 \mathrm{Li}_{2}(\theta)$

$$
=2 \operatorname{Li}_{2}\left(\theta^{9}\right)+3 \operatorname{Li}_{2}\left(\theta^{6}\right)+9 \operatorname{Li}_{2}\left(\theta^{2}\right) .
$$

The quantity $\theta_{2}=\theta^{2}$ satisfies $\theta_{2}^{3}+2 \theta_{2}^{2}+\theta_{2}=1$, and $(110 \mathrm{a}, \mathrm{b}, \mathrm{c})$ can be put in the form

$$
\text { (a) } \mathrm{Li}_{2}\left(\theta_{2}^{3}\right)=\mathrm{Li}_{2}\left(\theta_{2}^{2}\right)+4 \mathrm{Li}_{2}\left(\theta_{2}\right)+\frac{7}{4} \log ^{2} \theta_{2}-\pi^{2} / 3 \text {. }
$$

The quantity $\theta_{3}=\theta^{3}$ satisfies $\theta_{3}^{3}-3 \theta_{3}^{2}+4 \theta_{3}=1$, and (110f) can be put in the form

$$
\text { (b) } \mathrm{Li}_{2}\left(\theta_{3}^{6}\right)=2 \mathrm{Li}_{2}\left(\theta_{3}^{3}\right)+\frac{9}{2} \mathrm{Li}_{2}\left(\theta_{3}^{2}\right)-5 \mathrm{Li}_{2}\left(\theta_{3}\right)+\pi^{2} / 6-\frac{1}{3} \log ^{2} \theta_{3} .
$$

$5.14 \phi$.

5.14.1. The base equation is $1-\phi=\phi^{4}$ and leads to

$$
\phi^{2}+\phi^{4}+\phi^{5}=1, \quad \phi+\phi^{5}+\phi^{8}=1, \quad \phi+\phi^{3}-\phi^{7}=1
$$

with the following six independent relations:

(a) $\operatorname{Li}_{2}(\phi)+\operatorname{Li}_{2}\left(\phi^{4}\right)=\pi^{2} / 6-4 \log ^{2} \phi$,

(b) $2 \mathrm{Li}_{2}\left(\phi^{5}\right)=-5 \mathrm{Li}_{2}\left(\phi^{2}\right)+3 \mathrm{Li}_{2}(\phi)-5 \log ^{2} \phi$,

(c) $\operatorname{Li}_{2}\left(\phi^{6}\right)=2 \operatorname{Li}_{2}\left(\phi^{3}\right)-2 \operatorname{Li}_{2}\left(\phi^{4}\right)-\log ^{2} \phi$, or

(d) $\operatorname{Li}_{2}\left(\phi^{6}\right)=2 \operatorname{Li}_{2}\left(\phi^{3}\right)+2 \operatorname{Li}_{2}(\phi)-\pi^{2} / 3+7 \log ^{2} \phi$,

(e) $\operatorname{Li}_{2}\left(\phi^{9}\right)=3 \mathrm{Li}_{2}\left(\phi^{3}\right)-\frac{9}{2} \mathrm{Li}_{2}\left(\phi^{2}\right)+\frac{5}{2} \mathrm{Li}_{2}(\phi)-\pi^{2} / 6+\frac{5}{2} \log ^{2} \phi$,

(f) $\operatorname{Li}_{2}\left(\phi^{14}\right)=2 \mathrm{Li}_{2}\left(\phi^{7}\right)-7 \mathrm{Li}_{2}\left(\phi^{2}\right)+2 \mathrm{Li}_{2}(\phi)+\pi^{2} / 6-6 \log ^{2} \phi$,

(g) $\quad \mathrm{Li}_{2}\left(\phi^{24}\right)=2 \mathrm{Li}_{2}\left(\phi^{12}\right)+3 \mathrm{Li}_{2}\left(\phi^{8}\right)+4 \mathrm{Li}_{2}\left(\phi^{6}\right)-8 \mathrm{Li}_{2}\left(\phi^{3}\right)$

$$
-12 \operatorname{Li}_{2}\left(\phi^{2}\right)+5 \operatorname{Li}_{2}(\phi)+\pi^{2} / 6-2 \log ^{2} \phi \text {. }
$$


5.14.2. With $\phi_{2}=\phi^{2}, \phi_{2}+2 \phi_{2}^{2}-\phi_{2}^{4}=1$ we get, from (112),

$\mathrm{Li}_{2}\left(\phi_{2}^{12}\right)=2 \mathrm{Li}_{2}\left(\phi_{2}^{6}\right)+3 \mathrm{Li}_{2}\left(\phi_{2}^{4}\right)-7 \mathrm{Li}_{2}\left(\phi_{2}^{2}\right)-12 \mathrm{Li}_{2}\left(\phi_{2}\right)+\pi^{2}-\frac{15}{2} \log ^{2} \phi_{2}$.

$5.15 \psi$

5.15.1. The base equation is $\psi^{3}+\psi^{4}=1$, leading to

(114) $\psi^{4}+\psi^{6}+\psi^{7}=1, \quad \psi^{3}+\psi^{7}+\psi^{8}=1, \quad \psi+\psi^{3}-\psi^{5}=1$.

The equations, with six independent relations, are

(a) $2 \mathrm{Li}_{2}\left(\psi^{3}\right)=\mathrm{Li}_{2}\left(\psi^{2}\right)-2 \mathrm{Li}_{2}(\psi)+\pi^{2} / 3-15 \log ^{2} \psi$,

(b) $2 \mathrm{Li}_{2}\left(\psi^{4}\right)=-\mathrm{Li}_{2}\left(\psi^{2}\right)+2 \mathrm{Li}_{2}(\psi)-9 \log ^{2} \psi$, or

(c) $\operatorname{Li}_{2}\left(\psi^{3}\right)+\operatorname{Li}_{2}\left(\psi^{4}\right)=\pi^{2} / 6-12 \log ^{2} \psi$,

(d) $\operatorname{Li}_{2}\left(\psi^{7}\right)+3 \mathrm{Li}_{2}\left(\psi^{2}\right)+\mathbf{L i}_{2}(\psi)=\pi^{2} / 2-26 \log ^{2} \psi$, or

(e) $\operatorname{Li}_{2}\left(\psi^{7}\right)+8 \mathrm{Li}_{2}\left(\psi^{4}\right)+7 \mathrm{Li}_{2}\left(\psi^{2}\right)-7 \mathrm{Li}_{2}(\psi)=\pi^{2} / 2-62 \log ^{2} \psi$,

(f) $\quad \mathrm{Li}_{2}\left(\psi^{10}\right)=2 \mathrm{Li}_{2}\left(\psi^{5}\right)-\frac{7}{2} \mathrm{Li}_{2}\left(\psi^{2}\right)-3 \mathrm{Li}_{2}(\psi)+2 \pi^{2} / 3-\frac{49}{2} \log ^{2} \psi$, or

(g) $\quad \operatorname{Li}_{2}\left(\psi^{10}\right)=2 \operatorname{Li}_{2}\left(\psi^{5}\right)+2 \operatorname{Li}_{2}\left(\psi^{4}\right)-\frac{5}{2} \operatorname{Li}_{2}\left(\psi^{2}\right)$

$$
-5 \operatorname{Li}_{2}(\psi)+2 \pi^{2} / 3-\frac{31}{2} \log ^{2} \psi,
$$

(h) $\quad \operatorname{Li}_{2}\left(\psi^{18}\right)=2 \operatorname{Li}_{2}\left(\psi^{9}\right)+3 \operatorname{Li}_{2}\left(\psi^{6}\right)-2 \operatorname{Li}_{2}\left(\psi^{3}\right)$

$$
-9 \operatorname{Li}_{2}\left(\psi^{2}\right)+5 \pi^{2} / 6-33 \log ^{2} \psi,
$$

(i) $\operatorname{Li}_{2}\left(\psi^{24}\right)=2 \operatorname{Li}_{2}\left(\psi^{12}\right)+3 \operatorname{Li}_{2}\left(\psi^{8}\right)+6 \operatorname{Li}_{2}\left(\psi^{3}\right)$

$$
-12 \operatorname{Li}_{2}\left(\psi^{2}\right)+2 \pi^{2} / 3-27 \log ^{2} \psi \text {. }
$$

5.15.2. With $\psi_{2}=\psi^{2}, \psi_{2}^{3}+2 \psi_{2}^{2}=1+\psi_{2}^{4}$, we get, from (115i)

$$
\begin{aligned}
\operatorname{Li}_{2}\left(\psi_{2}^{12}\right)= & 2 \mathrm{Li}_{2}\left(\psi_{2}^{6}\right)+3 \mathrm{Li}_{2}\left(\psi_{2}^{4}\right)-6 \mathrm{Li}_{2}\left(\psi_{2}^{2}\right) \\
& -12 \mathrm{Li}_{2}\left(\psi_{2}\right)+5 \pi^{2} / 3-\frac{99}{4} \log ^{2} \psi_{2} .
\end{aligned}
$$

$5.16 \xi$. The base equations are

$$
\xi+\xi^{3}+\xi^{5}=1, \quad \xi+\xi^{2}-\xi^{7}=1
$$


leading to four independent equations

(a) $3 \mathrm{Li}_{2}\left(\xi^{5}\right)=\mathrm{Li}_{2}\left(\xi^{4}\right)-2 \mathrm{Li}_{2}\left(\xi^{3}\right)$

$$
+\mathrm{Li}_{2}\left(\xi^{2}\right)-\mathrm{Li}_{2}(\xi)+\pi^{2} / 6-3 \log ^{2} \xi,
$$

(b) $\operatorname{Li}_{2}\left(\xi^{6}\right)=2 \mathrm{Li}_{2}\left(\xi^{5}\right)+2 \mathrm{Li}_{2}\left(\xi^{3}\right)+2 \mathrm{Li}_{2}(\xi)-\pi^{2} / 3+5 \log ^{2} \xi$,

(c) $\mathrm{Li}_{2}\left(\xi^{8}\right)=\mathrm{Li}_{2}\left(\xi^{4}\right)+2 \mathrm{Li}_{2}\left(\xi^{3}\right)+3 \mathrm{Li}_{2}\left(\xi^{2}\right)-\pi^{2} / 3+6 \log ^{2} \xi$,

(d) $\mathrm{Li}_{2}\left(\xi^{14}\right)=2 \mathrm{Li}_{2}\left(\xi^{7}\right)+2 \mathrm{Li}_{2}\left(\xi^{5}\right)$

$$
+2 \mathrm{Li}_{2}\left(\xi^{3}\right)+\mathrm{Li}_{2}\left(\xi^{2}\right)-\pi^{2} / 3+7 \log ^{2} \xi .
$$

$5.17 \omega=\left(\frac{1}{2}(1+\sqrt{23 / 27})\right)^{2 / 3}+\left(\frac{1}{2}(1+\sqrt{23 / 27})\right)^{2 / 3}-\frac{1}{3}$.

5.17.1. The base equation is $1-\omega=\omega^{5}$, leading also to $1-\omega^{2}=\omega^{3}$ and $(1-\omega) /(1+\omega)=\omega^{7}$. Thus $\omega$ lies at the confluence of all the families discussed in Section 3, and has, by consequence, an extraordinarily rich range of results. Three-term equations satisfied by $\omega$ are

$$
\begin{array}{lll}
\omega+\omega^{7}+\omega^{8}=1, & \omega^{2}+\omega^{5}+\omega^{6}=1 ; & \omega+\omega^{6}+\omega^{10}=1, \\
\omega^{3}+\omega^{4}+\omega^{5}=1, & \omega+\omega^{3}-\omega^{6}=1, & \omega+\omega^{2}-\omega^{4}=1, \\
\omega^{2}+\omega^{2}-\omega^{7}=1, & \omega^{2}+\omega^{4}+\omega^{8}=1, & \omega^{3}+\omega^{3}+\omega^{7}=1, \\
& \omega+\omega^{4}-\omega^{9}=1 .
\end{array}
$$

There are considerable redundancies, but ten independent results exist, which can be expressed as follows:

(a) $\mathrm{Li}_{2}(\omega)+\mathrm{Li}_{2}\left(\omega^{5}\right)=\pi^{2} / 6-5 \log ^{2} \omega$,

(b) $\mathrm{Li}_{2}\left(\omega^{2}\right)+\mathrm{Li}_{2}\left(\omega^{3}\right)=\pi^{2} / 6-6 \log ^{2} \omega$,

(c) $\mathrm{Li}_{2}\left(\omega^{2}\right)+2 \mathrm{Li}_{2}(\omega)=\pi^{2} / 3-8 \log ^{2} \omega$,

(d) $\operatorname{Li}_{2}\left(\omega^{8}\right)=2 \operatorname{Li}_{2}\left(\omega^{4}\right)-\operatorname{Li}_{2}\left(\omega^{2}\right)+\log ^{2} \omega$,

(e) $\operatorname{Li}_{2}\left(\omega^{12}\right)=2 \mathrm{Li}_{2}\left(\omega^{6}\right)+3 \mathrm{Li}_{2}\left(\omega^{4}\right)+4 \mathrm{Li}_{2}\left(\omega^{3}\right)+6 \mathrm{Li}_{2}\left(\omega^{2}\right)$

$$
+2 \operatorname{Li}_{2}(\omega)-4 \pi^{2} / 3+47 \log ^{2} \omega \text {, }
$$

(f) $\mathrm{Li}_{2}\left(\omega^{14}\right)=4 \mathrm{Li}_{2}\left(\omega^{7}\right)-\mathrm{Li}_{2}\left(\omega^{2}\right)+4 \mathrm{Li}_{2}(\omega)-\frac{1}{2} \pi^{2}+14 \log ^{2} \omega$,

(g) $\operatorname{Li}_{2}\left(\omega^{18}\right)=2 \operatorname{Li}_{2}\left(\omega^{9}\right)+3 \mathrm{Li}_{2}\left(\omega^{6}\right)+6 \mathrm{Li}_{2}\left(\omega^{3}\right)+9 \operatorname{Li}_{2}\left(\omega^{2}\right)+2 \operatorname{Li}_{2}(\omega)$

$$
-5 \pi^{2} / 3+59 \log ^{2} \omega
$$

(h) $\operatorname{Li}_{2}\left(\omega^{20}\right)=2 \operatorname{Li}_{2}\left(\omega^{10}\right)+4 \operatorname{Li}_{2}\left(\omega^{5}\right)+10 \operatorname{Li}_{2}\left(\omega^{4}\right)$

$$
-10 \operatorname{Li}_{2}\left(\omega^{2}\right)-10 \mathrm{Li}_{2}(\omega)+4 \pi^{2} / 3-15 \log ^{2} \omega,
$$

(i) $\quad \mathrm{Li}_{2}\left(\omega^{28}\right)=2 \mathrm{Li}_{2}\left(\omega^{14}\right)+4 \mathrm{Li}_{2}\left(\omega^{7}\right)+7 \mathrm{Li}_{2}\left(\omega^{4}\right)$

$$
-6 \mathrm{Li}_{2}\left(\omega^{2}\right)-4 \mathrm{Li}_{2}(\omega)+\pi^{2} / 2 \text {, }
$$


(j) $\quad \mathrm{Li}_{2}\left(\omega^{30}\right)=2 \mathrm{Li}_{2}\left(\omega^{15}\right)+3 \mathrm{Li}_{2}\left(\omega^{10}\right)$

$$
\begin{aligned}
& +5 \operatorname{Li}_{2}\left(\omega^{6}\right)+6 \operatorname{Li}_{2}\left(\omega^{5}\right)+10 \operatorname{Li}_{2}\left(\omega^{3}\right) \\
& +15 \operatorname{Li}_{2}\left(\omega^{2}\right)+14 \operatorname{Li}_{2}(\omega)-13 \pi^{2} / 3+140 \log ^{2} \omega .
\end{aligned}
$$

5.17.2. Several equations for $\omega_{2}=\omega^{2}$, satisfying

$$
2 \omega_{2}-\omega_{2}^{2}+\omega_{2}^{3}=1
$$

can be found from (120). They are

(a) $\operatorname{Li}_{2}\left(\omega_{2}^{4}\right)=2 \operatorname{Li}_{2}\left(\omega_{2}^{2}\right)-\mathrm{Li}_{2}\left(\omega_{2}\right)+\frac{1}{4} \log ^{2} \omega_{2}$,

(b) $\operatorname{Li}_{2}\left(\omega_{2}^{6}\right)=2 \operatorname{Li}_{2}\left(\omega_{2}^{3}\right)+3 \operatorname{Li}_{2}\left(\omega_{2}^{2}\right)+\operatorname{Li}_{2}\left(\omega_{2}\right)-\pi^{2} / 3+\frac{15}{4} \log ^{2} \omega_{2}$,

(c) $\operatorname{Li}_{2}\left(\omega_{2}^{10}\right)=2 \operatorname{Li}_{2}\left(\omega_{2}^{5}\right)+10 \operatorname{Li}_{2}\left(\omega_{2}^{2}\right)-3 \operatorname{Li}_{2}\left(\omega_{2}\right)-\pi^{2} / 3+\frac{21}{4} \log ^{2} \omega_{2}$,

(d) $\operatorname{Li}_{2}\left(\omega_{2}^{14}\right)=3 \mathrm{Li}_{2}\left(\omega_{2}^{7}\right)+7 \mathrm{Li}_{2}\left(\omega_{2}^{2}\right)-\mathrm{Li}_{2}\left(\omega_{2}\right)-\pi^{2} / 3+\frac{9}{2} \log ^{2} \omega_{2}$.

5.17.3. There is one equation for $\omega_{3}=\omega^{3}$, which satisfies

$$
3 \omega_{3}-2 \omega_{3}^{2}+\omega_{3}^{3}=1 \text {. }
$$

It can be written

$$
\operatorname{Li}_{2}\left(\omega_{3}^{6}\right)=2 \operatorname{Li}_{2}\left(\omega_{3}^{3}\right)+3 \operatorname{Li}_{2}\left(\omega_{3}^{2}\right)-2 \operatorname{Li}_{2}\left(\omega_{3}\right)+\frac{1}{3} \log ^{2} \omega_{3} .
$$

$5.18 \Omega=\frac{1}{2}\left(1+3^{1 / 2}-12^{1 / 4}\right)=\sqrt{1+\cos (\pi / 6)}-\sqrt{\cos (\pi / 6)}$. The base equation comes from (138) with $p_{1}=2, p_{2}=p_{3}=p_{4}=p_{5}=1$, and is

$$
\Omega^{6}-3 \Omega^{4}-4 \Omega^{3}-3 \Omega^{2}+1=0 .
$$

This expression factorizes into $(\Omega+1)\left[\Omega^{2}-(1+\sqrt{3}) \Omega+1\right]\left[\Omega^{2}-(1-\sqrt{3}) \Omega+1\right]$ giving the single real root between 0 and 1 shown above. The resulting equation is

$$
\frac{3}{2} \operatorname{Li}_{2}\left(\Omega^{4}\right)-2 \operatorname{Li}_{2}\left(\Omega^{3}\right)-3 \operatorname{Li}_{2}\left(\Omega^{2}\right)-6 \operatorname{Li}_{2}(\Omega)=-7 \pi^{2} / 12+3 \log ^{2} \Omega
$$

\section{Clausen's function and multi-variable equations}

As indicated at the end of Section 3, the exponent form of the dilogarithm functional equation has a close affinity with the equation for Clausen's function [3], and this is exploited in the present analysis. 
6.1 The imaginary part of the dilogarithm. Clausen's function, for real angle $a$, is defined by the series

$$
\mathrm{Cl}_{2}(a)=\sum_{1}^{\infty} \frac{\sin n a}{n^{2}}
$$

or by the integral

$$
\mathrm{Cl}_{2}(a)=-\int_{0}^{a} \log ^{2}\left(2 \sin \frac{1}{2} x\right) d x .
$$

It is usual to square $2 \sin \left(\frac{1}{2} x\right)$ and take half the value of the integral, so that the representation remains true for negative values of $a$.With suitable care in interpreting the logarithm, (128) can be used to expand the definition to complex values of $a$.

The connection with the dilogarithm comes from the relation

$$
\mathrm{Li}_{2}\left(e^{i a}\right)=\frac{\pi^{2}}{6}-\frac{a(2 \pi-a)}{4}+i \mathrm{Cl}_{2}(a), \quad 0 \leqslant a \leqslant 2 \pi .
$$

The interesting feature is that the imaginary part of $\mathrm{Li}_{2}\left(R e^{i a}\right)$ (but not the real part) can still be expressed by an equation like (129). This result is not immediately obvious, and requires a subsidiary variable $b$ defined by

$$
\tan b=R \sin a /(1-R \cos a) .
$$

The relation, due to Kummer [6], is

$$
\operatorname{Im~} \mathrm{Li}_{2}\left(R e^{i a}\right)=b \log ^{2} R+\frac{1}{2} \mathrm{Cl}_{2}(2 b)+\frac{1}{2} \mathrm{Cl}_{2}(2 a)-\frac{1}{2} \mathrm{Cl}_{2}(2 a+2 b) \text {. }
$$

6.2 Multi-variable equations. If we take a formula like (16), treat $x$ and $y$ as complex variables (so that four independent real quantities are involved), take the imaginary parts, and express the result in Clausen functions, we will obtain a formula for Clausen functions with four independent real variables involving $3 \times 5=15$ transcendental terms. This exercise was performed by Kummer [6], and later by Rogers [11], and leads to the following equation involving five variables $a_{n}, n=1$ to 5 , with a single relation between them.

$$
\sum_{1}^{5}\left[\mathrm{Cl}_{2}\left(2 a_{n}\right)-\mathrm{Cl}_{2}\left(2 a_{n+2}+2 a_{n+3}\right)+\mathrm{Cl}_{2}\left(2 a_{n+2}+2 a_{n+3}-2 a_{n}\right)\right]=0 .
$$

The connection between the $a_{n}$ can be written

$$
\prod_{1}^{5} e^{i 2 a_{n}}-\sum_{1}^{5} e^{i 2\left(a_{n+1}+a_{n+2}+a_{n+3}\right)}+\sum_{1}^{5} e^{i 2\left(a_{n+1}+a_{n+2}\right)}=1
$$

where $a_{n+5}$ is to be interpreted as $a_{n}$. 
What is interesting about this relation is that although it can give real $a_{n}$ solutions, it also has imaginary solutions. Thus, if we use (129) on (132) to reconvert the Clausen functions back to dilogarithms we finish up with a fifteen-term real dilogarithm equation with four independent real variables. If we define new quantities by $e^{i 2 a_{n}}=u^{p_{n}}$, this functional equation is

$$
\sum_{1}^{5}\left[\mathrm{Li}_{2}\left(u^{p_{n}}\right)-\mathrm{Li}_{2}\left(u^{p_{n+2}+p_{n+3}}\right)+\mathrm{Li}_{2}\left(u^{p_{n+2}+p_{n+3}-p_{n}}\right)\right]=\frac{5 \pi^{2}}{6}+P \log ^{2} u
$$

where $P=\Sigma_{1}^{5} p_{n}\left(p_{n+2}-\frac{1}{2} p_{n}\right)$, and the variables are connected by an eleven-term equation

$$
\prod_{1}^{5} u^{p_{n}}-\sum_{1}^{5} u^{p_{n+1}+p_{n+2}+p_{n+3}}+\sum_{1}^{5} u^{p_{n+1}+p_{n+2}}=1 .
$$

The affinity of (134) to (132) is immediately obvious and exemplifies the significance of the exponent form.

Rogers [11] has shown the existence of functional equations for the dilogarithm for $N$ independent variables, involving $N^{2}+1$ transcendental terms. It has long been thought impossible to derive them from Abel's equation. Four variables would require 17 terms; as against 15 in (134). The process used here thus leads to a more "compact" equation, and apparently can be continued, leading to an increase in the number of terms by a factor of 3 each time the number of variables is doubled.

6.3 Negative arguments. A slightly different form comes by writing $\pi+a$ for $a$ in (129);

$$
\mathrm{Li}_{2}\left(-e^{i a}\right)=\frac{\pi^{2}}{6}-\frac{\pi^{2}-a^{2}}{4}+i \mathrm{Cl}_{2}(\pi+a), \quad-\pi \leqslant a \leqslant \pi .
$$

The same method as that used in Section 6.2 leads to

$$
\sum_{1}^{5}\left[\mathrm{Li}_{2}\left(-u^{p_{n}}\right)-\mathrm{Li}_{2}\left(u^{p_{n+2}+p_{n+3}}\right)+\mathrm{Li}_{2}\left(-u^{p_{n+2}+p_{n+3}-p_{n}}\right)\right]=-\frac{2 \pi^{2}}{3}+P \log ^{2} u
$$

where $P$ is as in (134), and now

$$
1+\prod_{1}^{5} u^{p_{n}}=\sum_{1}^{5} u^{p_{n+1}+p_{n+2}}+\sum_{1}^{5} u^{p_{n+1}+p_{n+2}+p_{n+3}} .
$$

Equation (134) is valid at $u=1$, but (137) is not, since $u=1$ will not satisfy (138).

The special case $p_{n}=1$ gives $u=\rho^{2}$, with the result of (71a). The case $p_{n}=1$, except for $p_{1}=0$ gives $u=\chi$, and the result of (91). 
The case $p_{1}=2, p_{2}=p_{3}=p_{4}=p_{5}=1$ gives (125) and (126).

There is a slightly neater form of (137) and (138), coming from putting $p_{n_{n}}+p_{n+1}=q_{n}$ and $\Sigma_{1}^{5} q_{n}=2 s$. It is

$$
\sum_{m, n} \operatorname{Li}_{2}\left(-u^{s-q_{n}-q_{m}}\right)-\sum_{1}^{5} \operatorname{Li}_{2}\left(u^{q_{n}}\right)=-\frac{2 \pi^{2}}{3}+\frac{1}{2}\left(s^{2}-\sum_{1}^{5} q_{n}^{2}\right) \log ^{2} u
$$

where

$$
u^{s}+1=\sum_{1}^{5}\left(u^{q_{n}}+u^{s-q_{n}}\right) .
$$

In (139), the first summation is over all ten different pairs $q_{n}+q_{m}$ with $n \neq m$. It may be noted that (140), as exemplified by (125), is symmetrical around its central term.

Equation (139) is also highly symmetrical in the exponents $q_{n}$.

\section{Equation structure}

The following observations are all taken from the results of Section 5. In most cases only plausible generalizations are offered for consideration-analytic proofs are lacking at this time.

7.1 Algebraic and trigonometric bases. Apart from some overlap at the lower end, there seems to be a clear distinction between the two types. Equations like (59) indicate that $\pi^{2} / M$ is involved for trigonometric forms involving $\pi / M$, and since $\mathrm{Li}_{2}(1)=\pi^{2} / 6$ is often involved too, multiples of $\pi^{2} / 6 M$ are to be expected. Landen's and Watson's results for $M=5$ and 7 clearly show this, as does Loxton's formula for $M=9$. Thus the $\pi^{2} / 54$ in the presumptive forms (80b) and $(80 \mathrm{c})$ should cause no surprise. There is no equivalent of this feature for the nontrigonometric bases, and simple multiples of $\operatorname{Li}_{2}(-1)=-\pi^{2} / 12$ are all that is observed. On the other hand the coefficient of the logarithm sometimes, but not invariably, seems to be related to the index of the equation; for example 18 for $N=6,48$ for $N=18$ in (110c, $\mathrm{f}$ ), or 7 for $N=14$ in (118d), 140 for $N=30$ in $(120 j)$. From (139) we see that the index is indeed involved (in that equation), though not in a simple manner: and similarly for the equations of Section 3.

There is one other possible clue to the existence of these distinct classes. The base equations (74) and (79) each contain four terms (counting $3 \kappa^{2}$, for example, as three terms), that is, an even number of terms. But the equations (22), (27) and (133) all contain an odd number. This may account for the extreme difficulty (if not impossibility) of getting some of these formulae via Abel's equation. Of 
course, the eleven terms of (133) can be reduced if there is some mutual cancellation, but terms can only cancel in pairs, leaving the odd character of the equation unimpaired.

7.2 Factors. Some, but by no means all, of the results of Section 5 exhibit the feature mentioned in the introduction, whereby the exponents run through the factors of the index, with the coefficients being closely related to the complementary factors. Thus (115h) and (115i) for $\psi,(112 \mathrm{~d}$ to $\mathrm{g}$ ) for $\phi,(69)$ for $\rho,(85)$ for $\varepsilon$, (88) for $\delta,(110)$ for $\theta,(120)$ for $\omega$; and others. There is a sort of "rich zone" centred around $\rho$ and $\omega$ where this property is particularly exemplified, $\omega$ being especially copious in this regard. For example, (120h) for $N=20$ has factor/exponent pairs of $(2,10),(4,5)(10,4)(10,2),(10,1)$. These combinations can hardly be accidental. There are equations involving $N=14$ or 28 with pairs $(2,7)$, $(7,2),(7,4)$; and so on. This property may possibly be related to the factorization aspect discussed next, though this, too, lacks any analytic derivation at this time.

7.3 Factorization. It was mentioned in the introduction that Roger's $L$-function, defined by (3), sometimes suppressed the presence of logarithms in the formulae. For example, (14) becomes simply

$$
L(z)+L(1-z)=\pi^{2} / 6 .
$$

There is no obvious reason why this should be so in all cases. Thus (69e) for $\mathrm{Li}_{2}\left(\rho^{20}\right)$, an equation already devoid of logarithms, goes over into the same relation for $L\left(\rho^{20}\right)$. Equation (69d) for $\operatorname{Li}_{2}\left(\rho^{12}\right)$ goes over into a similar equation, also without logarithms. These examples are quite typical, and it is instructive to see how it comes about. Thus in (69e), if the logarithmic terms are all collected on the left, they become $-10 \log \rho \log R$ where

$$
R=\frac{1-\rho^{20}}{1-\rho^{10}} \cdot \frac{1-\rho^{2}}{\left(1-\rho^{4}\right)^{3}} .
$$

A little algebra, together with the relation $\rho+\rho^{2}=1$, readily reduces $R$ to unity. Similarly, in (69d) we get $-6 \log \rho \log R^{\prime}$ where

$$
R^{\prime}=\frac{1-\rho^{12}}{1-\rho^{6}} \cdot \frac{1-\rho^{2}}{1-\rho^{4}} \cdot \frac{1}{1-\rho^{3}} .
$$

There is a considerable amount of algebraic simplification, but it is necessary again to use $\rho+\rho^{2}=1$ to reduce $R^{\prime}$ to 1 .

The role of the initial algebraic simplification is not entirely clear. Obviously there is no obligation to use it-failure to do so simply makes the reduction that much more tedious. However, the mere existence of forms such as (142) or (143), without lots of fractional powers, is clearly dependent on the factor property of 
Section 7.2. If $15 \mathrm{Li}_{2}\left(\rho^{4}\right)$ had been, say, $14 \mathrm{Li}_{2}\left(\rho^{4}\right)$, the factor $\left(1-\rho^{4}\right)$ in (142) would have been raised to the power $14 / 5$ instead of 3 , and the simplification of factors would have been hindered. At the same time, it is also apparent that algebraic simplification alone is not enough; the equation for $\rho$ must also be used. Put differently, if the ultimate result (suppression of logarithmic terms) can be taken for granted, relations like (142) become equations to determine the base-though in most cases of needlessly high degree. The interesting question is "why are the logarithmic terms suppressed in this way?" The fact that they are is a matter of verification in each case, but there does not seem to be any identifiable reason why it should be so. It is also interesting to note that the one equation investigated where the logarithm does not appear as the square of the logarithm of the base, (85) for $\operatorname{Li}_{2}\left(\varepsilon^{6}\right)$, is also one for which the use of $L$-functions fails to suppress the logarithm, the neat factorization of (85) notwithstanding.

7.4 Extrapolations from Loxton's result. With $\kappa$ given by $(79 a)$ it is readily verified that

$$
\frac{\left(1-\kappa^{3}\right)}{(1-\kappa)\left(1-\kappa^{2}\right)^{2}}=\frac{1}{\kappa^{2}} .
$$

An equation of this general character, but arbitrary, cannot be expected to yield anything useful; but if (144) is "viable", then the considerations of the preceding section would indicate that the combinations of terms in (80a) should correspond to a genuine identity; and in fact it is easily reduced to Loxton's formula (77). The quantities $\lambda$ and $\mu$ of $(79 b, c)$ similarly satisfy

$$
\begin{aligned}
& \text { (a) } \frac{\left(1+\lambda^{3}\right)}{(1+\lambda)\left(1-\lambda^{2}\right)^{2}}=\frac{1}{\lambda^{2}}, \\
& \text { (b) } \frac{\left(1+\mu^{3}\right)}{(1+\mu)\left(1-\mu^{2}\right)^{2}}=1 .
\end{aligned}
$$

These structures correspond to $(80 \mathrm{~b}, \mathrm{c})$, or, alternatively, to $(81 \mathrm{~b}, \mathrm{c})$. One can perform similar manipulations for Watson's $\beta$ and $\gamma$, both of which yield valid equations. Therefore it would be surprising if (145) were not viable; the only feature then needing to be determined is the correct multiple of $\pi^{2} / 54$. This was found numerically, and there can be no doubt that $(80 \mathrm{~b}, \mathrm{c})$ are correct, even though an analytic derivation is lacking at this time. The role of the factorization property is crucial in this demonstration, but it, too, lacks any formal justification. The difficulty, as mentioned in the introduction, is that the equations do not possess any true variables, so verification by differentiation is not possible. 


\section{Concluding remarks}

The preceding analysis has brought a certain amount of order to what may have appeared as a number of disparate numerical formulae, and in providing many more examples it has exposed the problem of the inner structure of these relations. The factorization property has emerged as central to understanding the results, but lacks a formal explanation at this time. Also important is the realization that the dilogarithm arguments can be usefully put in exponent form, and the existence of a genuine four-variable equation derivable from Abel's equation opens up new possibilities of analysis. Finally, the prediction of some new results emphasizes the need to provide analytic means for handling these equations and may point to the need for a more profound understanding of the properties of the dilogarithm in the complex plane. The early results of Euler and Landen can now be seen to be the tip of an iceberg of unlimited extent.

\section{References}

[1] N. H. Abel, Oeuvres complètes, Vol. 2, p. 193.

[2] G. V. Chudnovsky, 'Padé approximations to the generalized hypergeometric functions', $J$. Math. Pures Appl. 58 (4) (1979), 445-476.

[3] T. Clausen, J Reine Angew. Math. 8 (1832), 298-300.

[4] H. S. M. Coxeter, 'The functions of Schläfli and Lobatschefsky', Quart J. Math. Oxford Ser. 6 (1935), 13-29.

[5] C. J. Hill, Specimen exercitii analytici etc., Lund, (1830) p. 9.

[6] E. E. Kummer, J. Reine Angew. Math. 21 (1840), 74-90.

[7] J. Landen, Mathematical memoirs I pp. 112-118 (1780).

[8] A. M. Legendre, Exercises de calcul intégral, Vol. 1 (1811), p. 247.

[9] L. Lewin, Polylogarithms and associated functions (Elsevier--North-Holland, New York, Oxford, 1981).

[10] B. Richmond and G. Szekeres, 'Some formulas related to dilogarithms, the zeta function and the Andrews-Gordon identities', J. Austral. Math. Soc. 31 (1981), 362-373.

[11] L. J. Rogers, 'On function sum theorems connected with the series $\sum_{1}^{\infty} x^{n} / n^{2}$, Proc. London Math. Soc. 4 (1907), 169-189.

[12] W. Schaeffer, J. Reine Angew. Math. 30 (1846), 288.

[13] W. Spence, An essay on logarithmic transcendents (London and Edinburgh, 1809).

[14] G. N. Watson, 'A note on Spence's logarithmic transcendent', Quart J. Math. Oxford Ser. 8 (1937), 39-42.

980 McIntire

Boulder, Colorado 80303

U.S.A. 